\title{
Women and Atrial Fibrillation
}

ANNABELLE VOLGMAN ${ }^{1}$, Emelia Benjamin ${ }^{2}$, Anne Curtis ${ }^{3}$, Margaret Fang ${ }^{4}$, Kathryn Lindley $^{5}$, Gerald Naccarelli ${ }^{6}$, Carl Pepine ${ }^{7}$, Odayme Quesada ${ }^{8}$, Marmar Vaseghi ${ }^{9}$, Albert Waldo $^{10}$, Nanette Wenger ${ }^{11}$, and Andrea Russo ${ }^{12}$

${ }^{1}$ Rush University Medical Center

${ }^{2}$ Boston University

${ }^{3}$ University at Buffalo

${ }^{4}$ University of California San Francisco

${ }^{5}$ Washington University in St Louis

${ }^{6}$ Penn State Heart and Vascular Institute

${ }^{7}$ University of Florida College of Medicine

${ }^{8}$ Christ Hospital

${ }^{9}$ UCLA Cardiac Arrhythmia Center

${ }^{10}$ Case Western Reserve University School of Medicine

${ }^{11}$ Emory University School of Medicine

${ }^{12}$ Cooper University Hospital

December 2, 2020

\begin{abstract}
Atrial fibrillation (AF) remains a growing problem in the United States and worldwide, imposing a high individual and health system burden, including increased resource consumption due to repeated hospitalizations, stroke, dementia, heart failure, and death. This comprehensive review summarizes the most recent data on sex-related differences in risks associated with AF. Women with AF have increased risk of stroke and death compared to men, and possible reasons for this disparity are explored. Women also continue to have worse symptoms and quality of life, and poorer outcomes with stroke prevention, as well as with rate and rhythm control management strategies. Many current rhythm control treatment strategies for AF, including cardioversion and ablation, are used less frequently in women as compared to men, whereas women are more likely to be treated with rate control strategies or anti-arrhythmic drugs. Sex differences should be considered in treating women with AF to improve outcomes and women and men should be offered the same interventions for AF. We need to improve the evidence base to understand if variation in utilization of rate and rhythm control management between men and women represents health inequities or appropriate clinical judgement.
\end{abstract}

\section{Hosted file}

Volgman Final JCE Women and Atrial Fibrillation.pdf available at https://authorea.com/users/ 380748/articles/496609-women-and-atrial-fibrillation 